\title{
Electron cloud development in the Proton Storage Ring and in the Spallation Neutron Source
}

\author{
M. T. F. Pivi* and M. A. Furman ${ }^{\dagger}$ \\ Center for Beam Physics, Accelerator and Fusion Research Division, Lawrence Berkeley National Laboratory, \\ MS 71-R0-259, Berkeley, California 94720
}

(Received 8 October 2002; published 5 March 2003)

\begin{abstract}
We have applied our simulation code POSINST to evaluate the contribution to the growth rate of the electron cloud instability in proton storage rings. In particular, we present here recent simulation results for the main features of the electron cloud in the storage ring of the Spallation Neutron Source at Oak Ridge, and updated results for the Proton Storage Ring at Los Alamos. A key ingredient in our model is a detailed description of the secondary electron emission process, including a refined model for the emitted energy spectrum, and for the three main components of the secondary yield, namely, the true secondary, rediffused and backscattered components.
\end{abstract}

DOI: 10.1103/PhysRevSTAB.6.034201

PACS numbers: 29.27.Bd, 79.20.Hx, 29.20.Dh

\section{INTRODUCTION}

The electron cloud effect may limit the performance of intense proton storage rings, causing a fast instability that may be responsible for proton losses and collective beam motion above a certain current threshold, accompanied by a large number of electrons. Electron cloud dedicated studies have been initiated at the Spallation Neutron Source (SNS) under construction at the Oak Ridge National Laboratory (ORNL). It is becoming progressively clear that the electron cloud effect plays an important role in the high-intensity instability which has been observed in the Proton Storage Ring (PSR) at the Los Alamos National Laboratory for more than 13 years. This instability is now believed to be due to the collective coupling between an electron cloud and the proton beam $[1,2]$. Such instability is a particular manifestation of the electron cloud effect (ECE) that has been observed or is expected at various other machines. In this article we present simulation results for the SNS and for PSR ring obtained with the ECE code that has been developed at LBNL over the past six years. In all results presented here, the proton beam is assumed to be a static distribution of given charge and shape moving on its nominal closed orbit, while the electrons are treated fully dynamically. Furthermore, we can infer details of the electron cloud in the vicinity of the proton beam, such as the neutralization factor, which is important for a selfconsistent treatment of the coupled $e-p$ problem in long, intense, proton bunches [3]. Current instability threshold, growth rate, and frequency spectrum are deferred to future studies.

Recently a comparative study of the ECE at various intense proton storage rings has been published [4] by means of simulations similar to those presented here.

\footnotetext{
*Present address: SLAC, P.O. Box 20450, Stanford, CA

94309.

Electronic address: mpivi@lbl.gov

${ }^{\dagger}$ Electronic address: mafurman@lbl.gov
}

Although our results for the PSR and SNS are in qualitative agreement with those in the comparative study, certain quantitative details are not, primarily owing to differences in the secondary emission model parameters we use in our studies (see our discussion below). Such parameters, in particular, the value of the secondary electron yield (SEY) at very low energy, deserve further attention if reliable quantitative predictions are to be achieved for the electron cloud density distribution and its time scales.

\section{PHYSICAL MODEL}

\section{A. Sources of electrons}

Electron sources may be classified into (1) electrons produced at the injection region stripping foil; (2) electrons produced by proton losses incident the vacuum chamber at grazing angles; (3) secondary electron emission process; and (4) electrons produced by residual gas ionization. The two main sources of electrons considered for proton storage rings at the SNS and the PSR are lost protons hitting the vacuum chamber walls and secondary emission from electrons hitting the walls (the electron cloud in the vicinity of the stripper foil is not modeled here). Although our code accommodates other sources of electrons, we have turned them off for the purposes of this article, as they are negligible compared to those above.

\section{B. Secondary emission process}

The SEY $\delta\left(E_{0}\right)$ and the corresponding emitted-electron energy spectrum $d \delta / d E\left(E_{0}=\right.$ incident electron energy, $E=$ emitted secondary energy) are represented by a model described in detail elsewhere [5]. The parameters related to the secondary emission process were obtained from detailed fits to the measured SEY of stainless steel [6]. The main SEY parameters are the energy $E_{\max }$ at which $\delta\left(E_{0}\right)$ is maximum, and the peak value itself, $\delta_{\max }=\delta\left(E_{\max }\right)$ (see Table I). For the results shown below, 
TABLE I. Simulation parameters for the PSR and SNS.

\begin{tabular}{lccc}
\hline \hline \multicolumn{1}{c}{ Parameter } & Symbol (unit) & PSR & SNS \\
\hline Ring parameters & & & \\
Proton beam energy & $B(\mathrm{GeV})$ & 1.735 & 1.9 \\
Dipole field & $B(\mathrm{~T})$ & 1.2 & 0.78 \\
Bunch population & $N_{p}\left(10^{13}\right)$ & 5 & 20.5 \\
Ring circumference & $C(\mathrm{~m})$ & 90 & 248 \\
Revolution period & $T(\mathrm{~ns})$ & 350 & 945 \\
Bunch length & $\tau_{b}(\mathrm{~ns})$ & 254 & 700 \\
Gaussian transverse beam size & $\sigma_{x}, \sigma_{y}(\mathrm{~mm})$ & 10,10 & $\ldots$ \\
Flat transverse beam size & $r_{x}, r_{y}(\mathrm{~mm})$ & $\ldots$ & 28,28 \\
Beam pipe semiaxes & $a, b(\mathrm{~cm})$ & 5,5 & 10,10 \\
Simulation parameters & & & \\
Proton loss rate & $p_{\text {loss }}\left(10^{-6}\right)$ & 4 & 1.1 \\
Proton-electron yield & $Y$ & 100 & 100 \\
No. kicks/bunch & $N_{k}$ & 1001 & 10001 \\
No. steps during gap & $N_{g}$ & 400 & 1000 \\
Maximum secondary yield & $\delta_{\max }$ & 2.0 & 2.0 \\
Energy at maximum yield & $E_{\max }(\mathrm{eV})$ & 300 & 250 \\
Yield low energy electron & $\delta(0)$ & 0.5 & 0.5 \\
Rediffused component & $P_{1, r}(\infty)$ & 0.74 & 0.2 \\
\hline \hline
\end{tabular}

we do take into account the elastic backscattered and rediffused components of the secondary emitted-electron energy spectrum $d \delta / d E$.

The conventional picture of secondary emission, which we base on various reviews of the subject [7-9], can be summarized as follows: when a steady current $I_{0}$ of electrons impinges on a surface, a certain portion $I_{e}$ is backscattered elastically while the rest penetrates into the material. Some of these electrons scatter from one or more atoms inside the material and are reflected back out. These are the so-called "rediffused" electrons, and we call the corresponding current $I_{r}$. The rest of the electrons interact in a more complicated way with the material and yield the so-called "true secondary electrons," whose current we call $I_{t s}$. The yields for each type of electron are defined by $\delta_{e}=I_{e} / I_{0}, \delta_{r}=I_{r} / I_{0}$, and $\delta_{t s}=I_{t s} / I_{0}$, so that the total SEY is

$$
\delta=\left(I_{e}+I_{r}+I_{t s}\right) / I_{0}=\delta_{e}+\delta_{r}+\delta_{t s} .
$$

Experimental data $[7,8]$ suggest that $\delta_{e}\left(E_{0}, \theta_{0}\right)$ and $\delta_{r}\left(E_{0}, \theta_{0}\right)$ at normal incidence $\left(\theta_{0}=0\right)$ are well characterized by empirical formulas such as

$$
\delta_{e}\left(E_{0}, 0\right)=P_{1, e}(\infty)+\left[\hat{P}_{1, e}-P_{1, e}(\infty)\right] e^{-\left(\left|E_{0}-\hat{E}_{e}\right| / W\right)^{p} / p},
$$

and

$$
\delta_{r}\left(E_{0}, 0\right)=P_{1, r}(\infty)\left[1-e^{-\left(E_{0} / E_{r}\right)^{r}}\right]
$$

while the energy dependence of $\delta_{t s}\left(E_{0}, 0\right)$ is well fit experimentally $[5,10]$ by an approximately universal [11] function of the form

034201-2

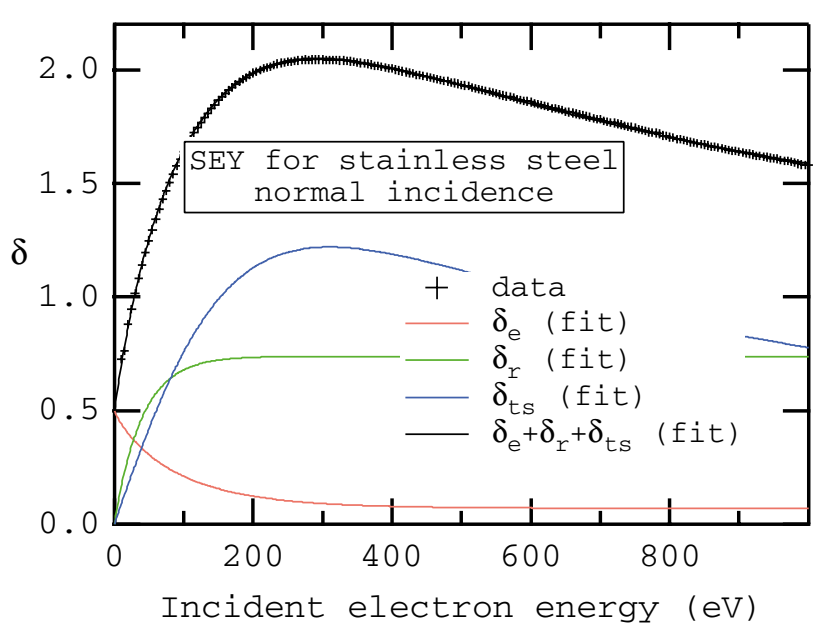

FIG. 1. (Color) The SEY for stainless steel for SLAC standard 304 rolled sheet, chemically etched and passivated but not conditioned. The parameters of the fit are listed in Table II. Data courtesy of R. Kirby.

$$
\delta_{t s}\left(E_{0}, 0\right)=\frac{s\left(E_{0} / \hat{E}_{t s}\right) \hat{\delta}_{t s}}{s-1+\left(E_{0} / \hat{E}_{t s}\right)^{s}}
$$

A sample fit to stainless steel data is shown in Fig. 1.

Simulation results are sensitive to details of the model for (1) the energy spectrum of the secondary emitted electrons [5] and (2) the backscattered electron component, in particular, to $\delta(0)$. The backscattered component typically becomes more important at low incident electron energies. To account for this behavior we have used a fit extrapolated from data for copper measured at CERN [12]. The value of $\delta\left(E_{0}\right)$ at incident electron

TABLE II. Main parameters of the model, typical values used for the SNS, titanium nitride coated stainless steel.

\begin{tabular}{cc}
\hline \hline Backscattered electrons & \\
$P_{1, e}(\infty)$ & 0.02 \\
$\hat{P}_{1, e}$ & 0.5 \\
$\hat{E}_{e}(\mathrm{eV})$ & 0 \\
$W(\mathrm{eV})$ & 60 \\
$p$ & 1 \\
Rediffused electrons & \\
$P_{1, r}(\infty)$ & 0.19 \\
$E_{r}(\mathrm{eV})$ & 0.04 \\
$r$ & 0.1 \\
True secondary electrons & \\
$\hat{E}_{t s}(\mathrm{eV})$ & 246 \\
$\hat{\delta}_{t s}$ & 1.8 \\
$s$ & 1.54 \\
Total SEY & \\
$\hat{E}_{t}(\mathrm{eV})$ & 250 \\
$\hat{\delta}_{t}$ & 2 \\
\hline \hline
\end{tabular}




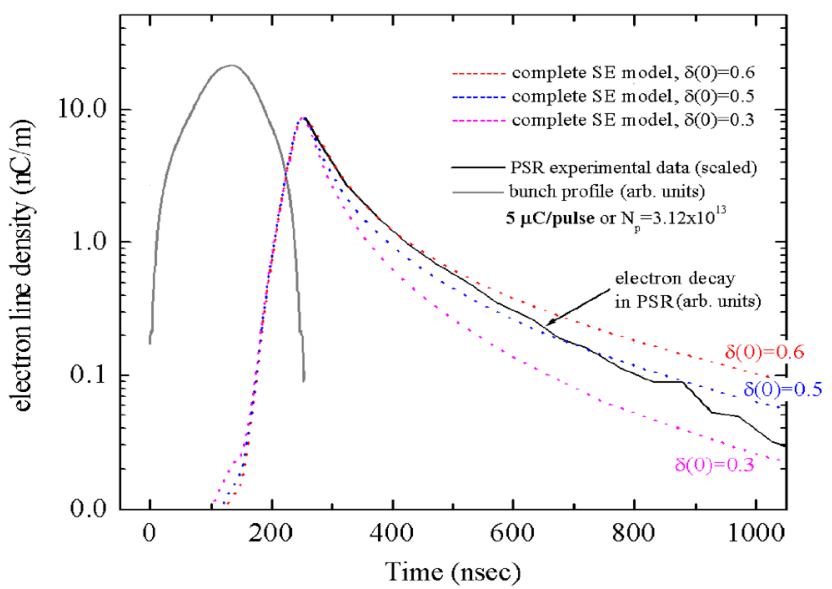

FIG. 2. (Color) Experimental data from PSR and simulated decay time of the electron cloud at the end of a beam pulse with bunch population $N_{p}=3.12 \times 10^{13}$ or $5 \mu \mathrm{C}$ intensity. Different SEY values for low energy electrons, $\delta(0)$, have been assumed in the simulation, with $\delta_{\max }=2$.

energies $E_{0}<10 \mathrm{eV}$ is an important parameter since it determines the electron survival rate in the gap. This quantity is difficult to measure experimentally and remains an uncertainty for the model. However, an indication may be given by the decay time of the electron cloud at the end of the beam pulse, which has been measured in the PSR [13]. A long exponential tail seen with $170 \mathrm{~ns}$ decay time may imply a high reflectivity for low energy electrons. In Fig. 2, we reproduce the passage of one single PSR beam assuming different values for $\delta(0)$. It is apparent that the experimental data implies $\delta(0) \lesssim 0.6$.

\section{Simulation model}

The beam-electron interaction and the twodimensional electron space-charge field used in the model are described in detail in Refs. $[10,14]$. The PSR and the

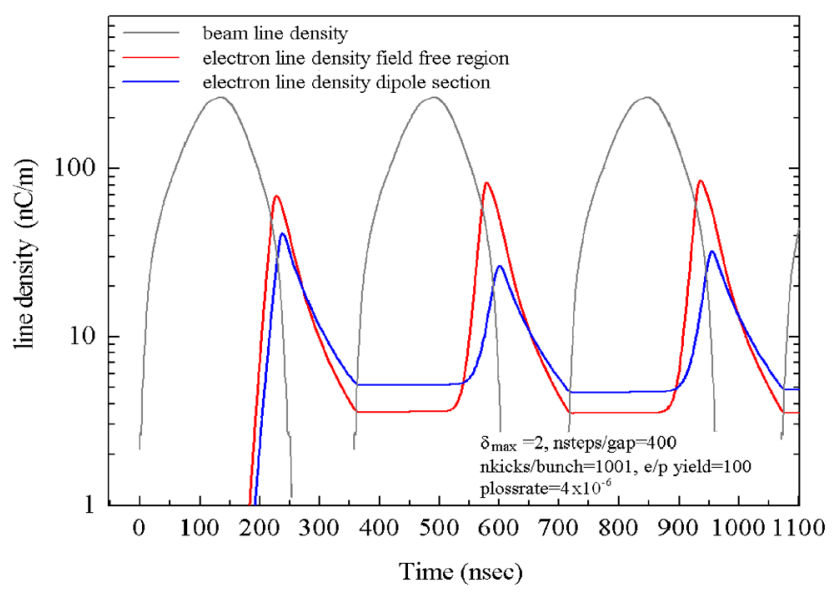

FIG. 3. (Color) Simulated electron density during the first bunch passages, in a PSR field-free region and a dipole section. The saturation level is reached after a few bunch passages.

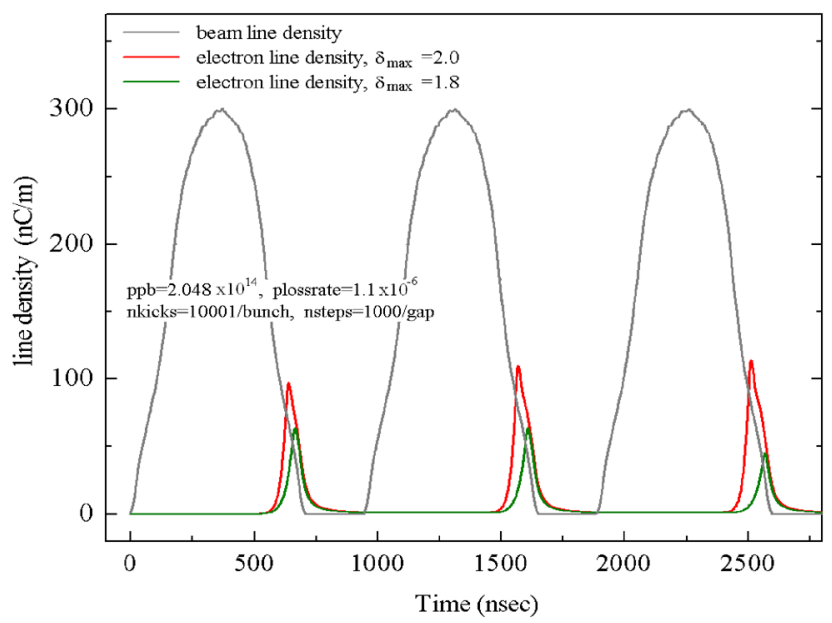

FIG. 4. (Color) Simulated electron density during the first bunch passages, in a SNS field-free region, assuming $\delta_{\max }=$ 2 and $\delta_{\max }=1.8$.

SNS rings store a single proton bunch of length $\tau_{b}$ followed by a gap of length $\tau_{g}$ with a typical current intensity profile shown in Figs. 3 and 4. A Gaussian transverse beam with rms sizes $\sigma_{x}=\sigma_{y}=10 \mathrm{~mm}$ and the actually measured longitudinal intensity profile are assumed for the PSR. The transverse beam distribution for the SNS is assumed to be constant with $r_{x}=r_{y}=28 \mathrm{~mm}$. The vacuum chamber is assumed to be a cylindrical perfectly conducting pipe. The number of electrons generated by lost protons hitting the vacuum chamber wall is $N_{p} \times$ $Y \times p_{\text {loss }}$ per turn for the whole ring, where $Y$ is the effective electron yield per lost proton, and $p_{\text {loss }}$ is the proton loss rate per turn for the whole ring per beam proton. The lost-proton time distribution is proportional to the instantaneous bunch intensity. The electrons are then simulated by macroparticles. The secondary electron mechanism adds to these a variable number of macroparticles, generated according to the SEY model mentioned above. The bunch is divided up into $N_{k}$ kicks, and the interbunch gap into $N_{g}$ intermediate steps. The image and space-charge forces are computed and applied at each slice in the bunch and each step in the gap. Making the approximation that the electron cloud density is longitudinally uniform, we compute the space-charge forces by means of a two-dimensional electric field. Typical parameter values are shown in Table I. These parameters imply $\delta(0)=0.5$, a choice based on Fig. 2; we assume this value for the following simulations presented here, unless stated otherwise.

\section{RESULTS AND DISCUSSIONS}

The possible amplification mechanism which may take place in long-beam storage rings is explained in Fig. 5. An electron present in the vacuum chamber before the bunch passage oscillates in the beam potential well. The oscillation amplitude most likely remains smaller than the chamber radius during the beam passage until the 


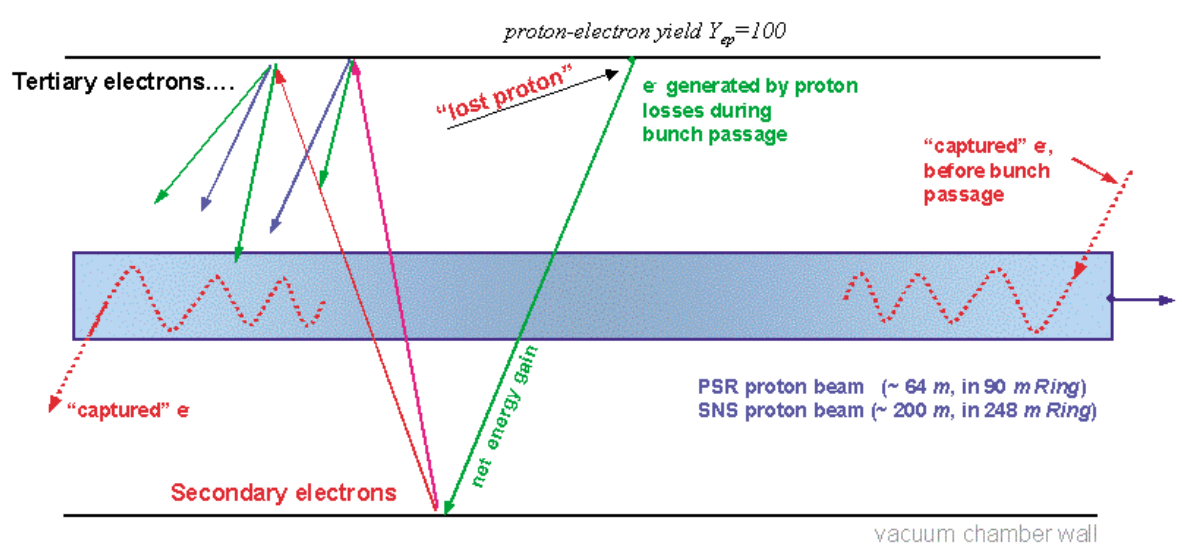

FIG. 5. (Color) Electron multiplication mechanism in long proton bunches.

electron is released towards the tail of the bunch. On the other hand, electrons generated at the wall by proton losses near the peak of the beam pulse are accelerated and decelerated by the beam potential and hit the opposite wall with a net energy gain, producing secondary electrons.

Electrons which survive the gap between two bunch passages will increase in number. The electrons gradually increase in number during successive bunch passages until, owing to the space-charge forces, a balance is reached between emitted and absorbed electrons (see also an animation of the PSR electron cloud dynamics during the beam passage in Ref. [15]).

The current and energy distribution of the electrons hitting the vacuum chamber wall have been measured with dedicated probes [16]. A typical measurement, obtained with the electron detector ED02X at the PSR, is shown in Fig. 6. It should be mentioned that surface conditioning occurred before the measurements were taken. Furthermore, in different sections of the ring, other electron detectors have measured a factor 10 higher electron wall currents. Figure 7 shows our simulation result, for which we assume unit detector efficiency and acceptance.

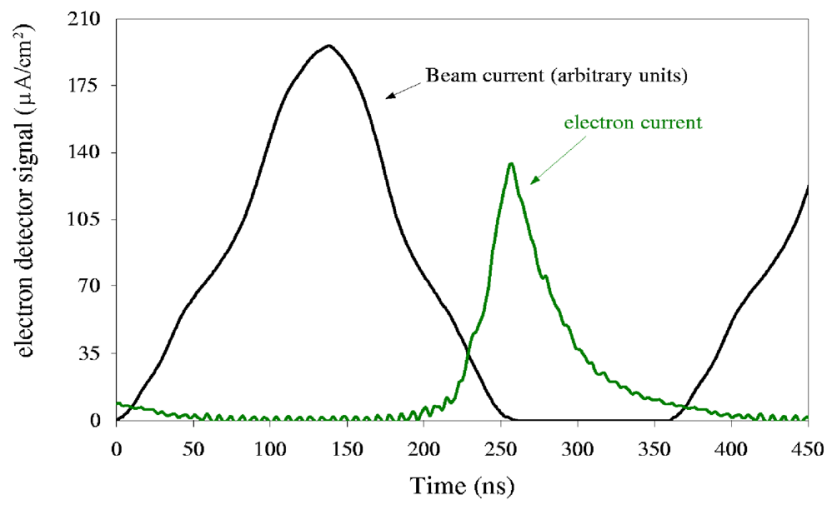

FIG. 6. (Color) Measured electron detector (ED02X, located at the injection region) signal at the PSR.
By applying a negative potential on the second grid of the electron probe it is possible to select the electrons with an energy sufficient to pass the repeller voltage and thus measure their integrated energy distribution. A typical measurement of the cumulative energy spectrum is shown in Fig. 8; our simulation result is shown in Fig. 9. The measured peak of the electron distribution at the wall (obtained by differentiating the cumulative spectrum with respect to energy) is at $\sim 240 \mathrm{eV}$ in rough agreement with the corresponding simulated number, $\sim 180 \mathrm{eV}$. Furthermore, also in the case of the energy spectrum measurements, detectors located in different sections of the ring have measured higher electron current signals.

The buildup of the electron cloud in a PSR field-free region and a dipole section during the passage of the beam is shown in Fig. 3. The saturation level in the PSR is reached after a few bunch passages, when assuming $\delta_{\max }=2$. The estimated peak number of electrons in a field-free region is $\sim 75 \mathrm{nC} / \mathrm{m}$ or $6 \times 10^{7} e / \mathrm{cm}^{3}$. When assuming $\delta(0) \simeq 0.5$ the simulated electron density in the PSR increases by a factor $\sim 3$ relative to the $\delta(0) \simeq 0.1$ case (refer to our previous results for the PSR, [17]).

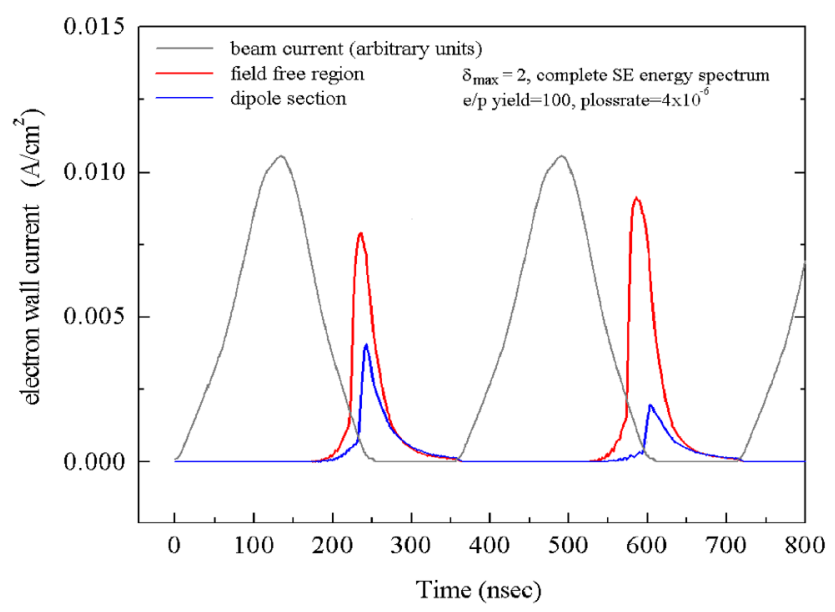

FIG. 7. (Color) Simulated current of electrons hitting the surface of the vacuum chamber during the first bunch passages, in a PSR field-free region and a dipole section.

034201-4 


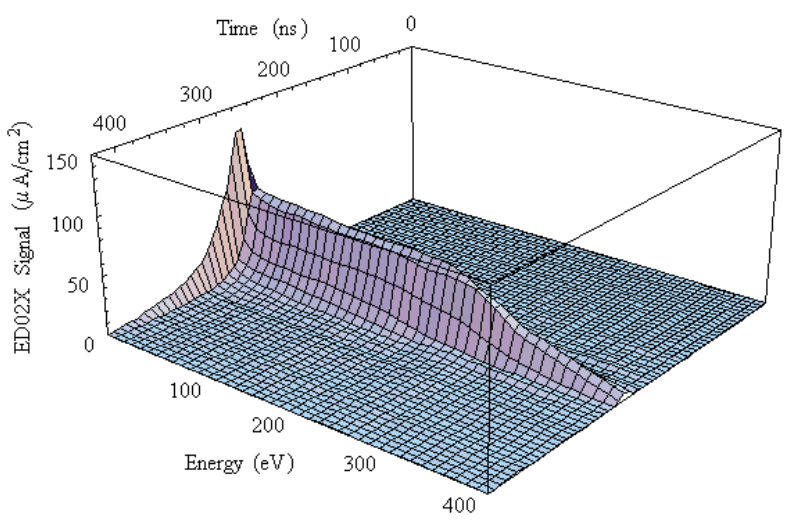

FIG. 8. (Color) Measured cumulative energy spectrum of the electrons hitting the wall as a function of time in the PSR (the origin of time corresponds to the passage of the head of the bunch).

These are examples of strong parameter sensitivity that calls for further experimental investigations. The neutralization factor or fractional charge neutralization, ratio $e / p$, during a bunch passage is shown in Fig. 10.

The SNS beam pipe chamber will be coated with TiN. Recent measurements of an as-received sample of the TiN-coated stainless steel SNS vacuum chamber have shown a secondary electron yield $\delta_{\max }=2 \pm 0.2$ [18]. Furthermore, recent secondary yield measurements of TiN-coated samples show $\delta_{\max }=1.9$ [19]. However, once well conditioned [6], TiN-coated samples can reach $\delta_{\max }$ as low as 1.1. Even if the peak value reached is in the more realistic range 1.3-1.5, simulations show significantly reduced electron cloud effects [20], which strongly suggests the use of such a coating as a mitigating mechanism.

Because of the large electron multiplication, we have used a relatively small number of macroparticles generated per bunch passage, which leads, nevertheless, to reasonably stable results in term of the turn-by-turn elec-

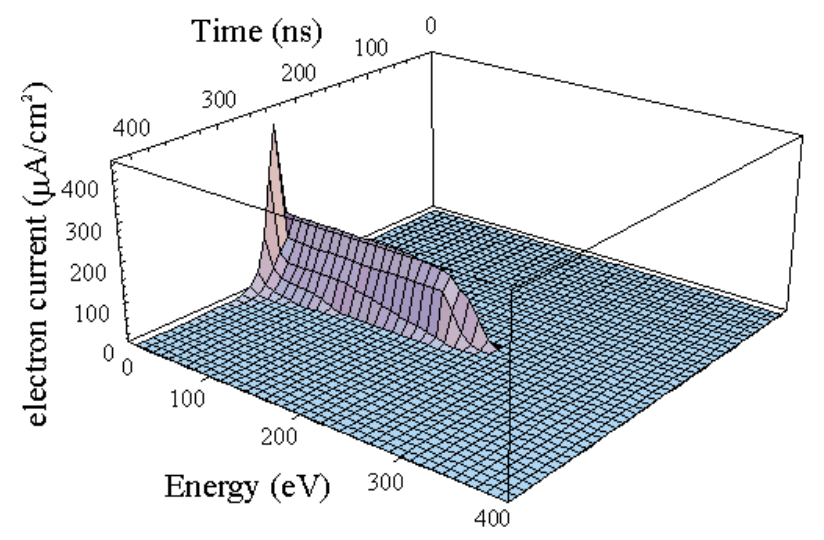

FIG. 9. (Color) Simulated cumulative energy spectrum of the electrons hitting the wall as a function of time in the PSR (the origin of time corresponds to the passage of the head of the bunch).

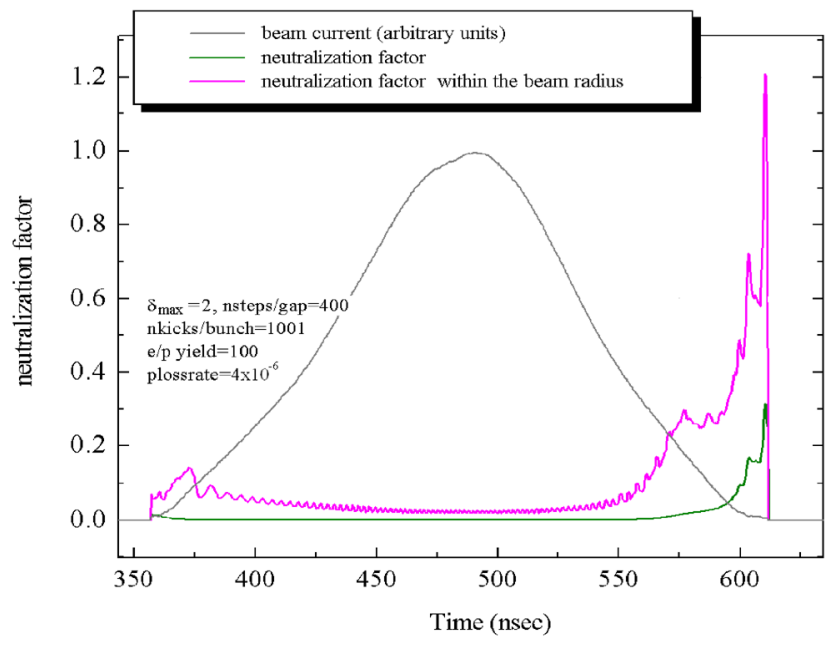

FIG. 10. (Color) Simulated electron neutralization factor in a PSR field-free region. The fractional charge neutralization computed within the beam radius region is $2 \%$ during the bunch passage, and it exceeds $20 \%$ at the tail of the bunch.

tron density. The amplification factor per macroparticle may exceed $10^{4}$ during a single bunch passage when $\delta_{\max }=2$. Simulation results for the SNS obtained with a different code [21] show a qualitative agreement with our results, although they yield a lower estimated electron density at this SEY value [18].

We assume in these simulations that beam particles are lost at the rate of $1.1 \times 10^{-6}$ protons per stored proton per turn, as expected in the SNS ring. For $\delta_{\max }=2$ and 1.8, the buildup of the electron cloud during the first few bunch passages is shown in Fig. 4 and the electron density computed within the beam radius region is shown in Fig. 11. A total line density of $100 \mathrm{nC} / \mathrm{m}$ and a line density within the beam radius region exceeding $10 \mathrm{nC} / \mathrm{m}$ are expected in a field-free region. These imply neutralization factors as shown in Fig. 12. In particular the neutralization factor during the bunch passage is $1 \%$, and most of the electrons are contained in the beam radius region.

A 2D particle density histogram of the $x-y$ phase space, averaged over time, is shown in Fig. 13. The electrons are localized most of the time in the inner region of the beam.

The electric field from the cloud leads to a neutralization tune shift which adds to the direct space-charge tune shift. If we make the approximation that the transverse electron cloud density is uniform within the bunch, a simple estimate can be obtained. Furthermore, the kinetic energy does not exceed few $\mathrm{keV}$, hence the electrons can be considered nonrelativistic. Thus the force on any given proton due to the electron cloud is approximately transverse. The tune shift due to electron neutralization may be estimated, for example, for particles in the beam at $25 \%$ of the peak intensity where the neutralization factor is $f=0.13$, by

$$
\Delta Q_{e c}=-0.25 f \gamma^{2} \Delta Q_{s c} \simeq-0.13 \Delta Q_{s c}=0.026,
$$




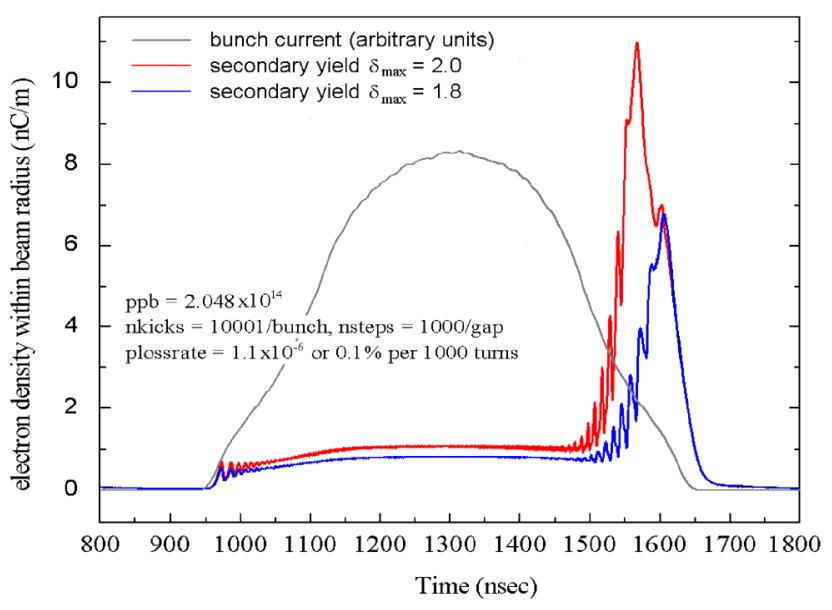

FIG. 11. (Color) Simulated electron density computed within the beam radius, in a SNS field-free region, assuming $\delta_{\max }=2$ and $\delta_{\max }=1.8$.

where $\Delta Q_{s c}=-0.2$ is the space-charge tune shift and $\gamma=2.066$ is the usual relativistic factor of the beam.

The possible amplification mechanism which may take place in long-beam storage rings suggests interesting considerations. Electrons generated at the wall by proton losses near the peak of the beam pulse are accelerated and decelerated by the beam potential and hit the opposite wall with a net energy gain, producing secondary electrons. Many generations of secondary electrons may occur during a single bunch passage leading to a high electron cloud density owing to trailing-edge multipacting. In order to verify this assumption, in the simulations, we have artificially truncated the tail of the bunch, while maintaining the same integrated beam charge. In particular, Fig. 14 shows the modified SNS beam current profile, compared to the nominal beam current profile. The effect of the modification of the

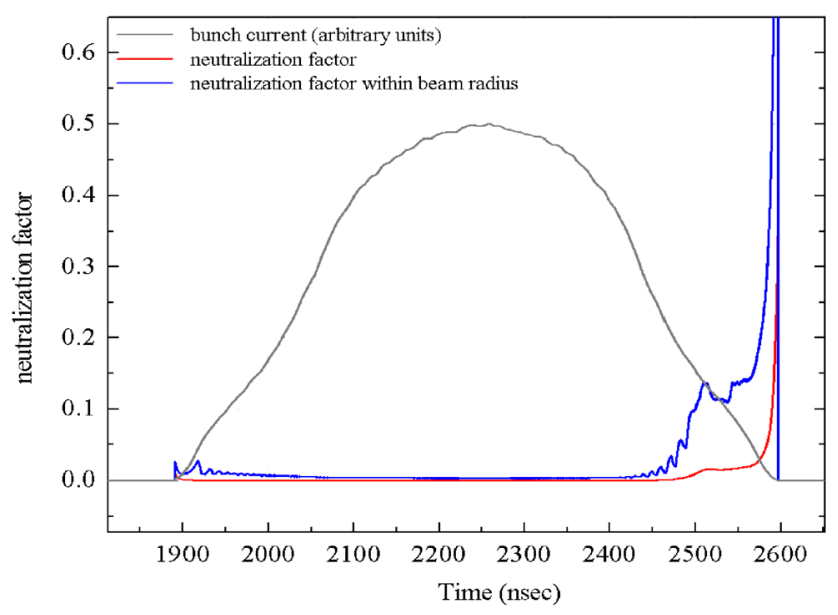

FIG. 12. (Color) Simulated electron neutralization factor in a SNS field-free region. The fractional charge neutralization computed within the beam radius region is $\sim 1 \%$ during the bunch passage, and it exceeds $10 \%$ at the tail of the bunch.

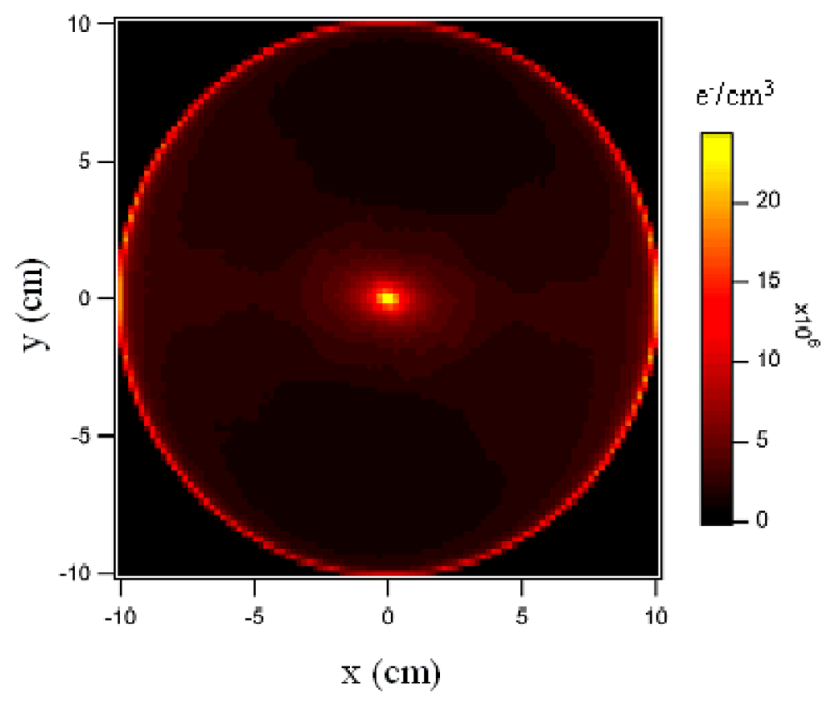

FIG. 13. (Color) Image of the time-averaged density of the electron cloud in an SNS field-free region. The higher-density horizontal "stripe" is due to a statistical fluctuation caused by strong multipacting.

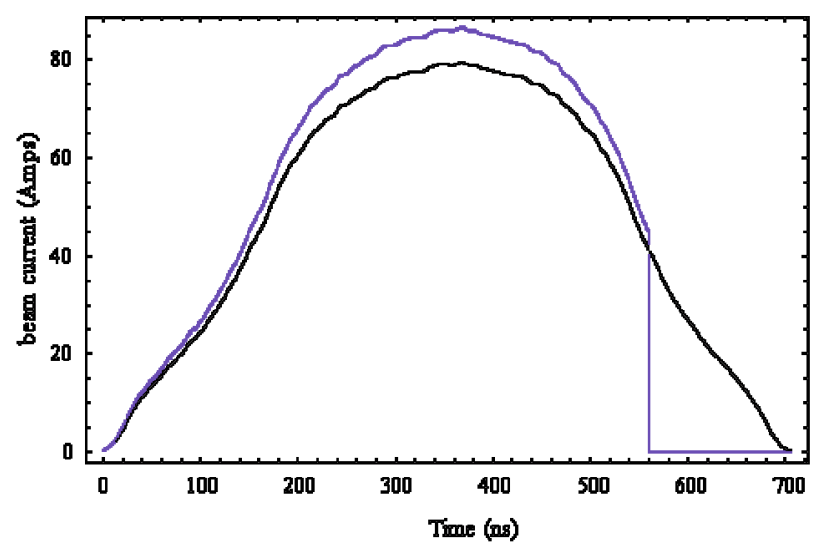

FIG. 14. (Color) SNS beam current profile cut at $560 \mathrm{~ns}$, compared to the nominal beam profile.

beam profile on the formation of the electron cloud is shown in Fig. 15. The density of the electron cloud decreases dramatically as the tail of the beam is progressively truncated owing to the breaking of the multipacting resonance condition. A reduction of the beam head profile has the opposite effect of increasing the electron cloud density. Simulation results for the PSR have shown a similar behavior. It seems interesting, therefore, to determine the extent to which such a tailoring of the bunch profile is feasible in practice.

\section{CONCLUSIONS}

We have presented electron cloud simulations for PSR and for the SNS. In the case of the PSR, we have compared our simulation results with measurements of the current and energy distribution of the electrons hitting the vacuum chamber wall. When considering proton 


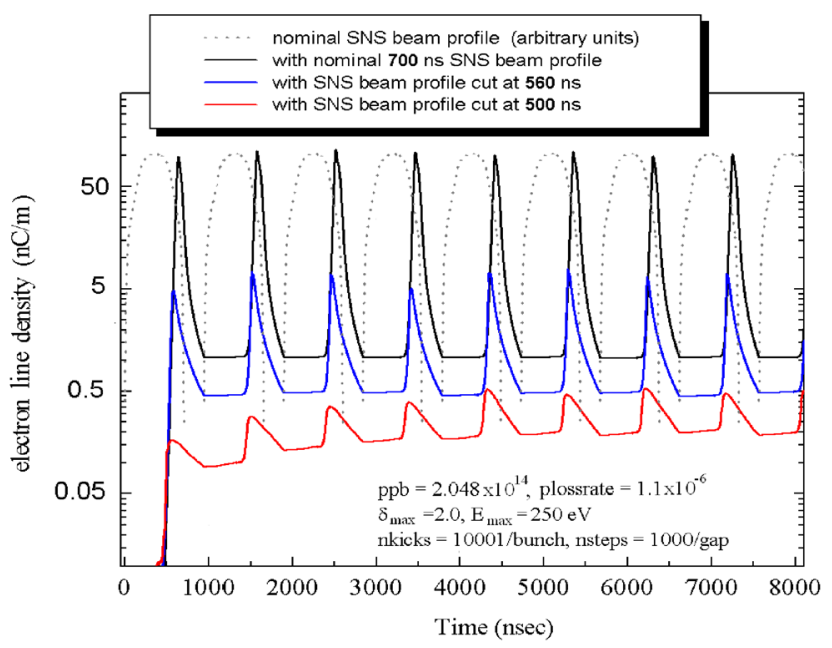

FIG. 15. (Color) Simulated electron density in an SNS fieldfree region, assuming $\delta_{\max }=2$ and $N_{p}=2.05 \times 10^{14}$. We have artificially truncated the tail of the bunch, while maintaining the same integrated beam charge. The density of the electron cloud decreases as the tail of the beam is progressively reduced.

losses of $10^{-6}$ and $\delta_{\max }=2$, a line density of $\geq$ $100 \mathrm{nC} / \mathrm{m}$ should be expected in an SNS field-free region, with a density exceeding $10 \mathrm{nC} / \mathrm{m}$ within the beam radius region. Although the neutralization factor may exceed $10 \%$ near the tail of the beam, the resulting electron cloud tune shift is moderate. Linear stability studies and current threshold estimates are deferred to a separate publication [18].

\section{ACKNOWLEDGMENTS}

We are particularly indebted to our colleagues of the PSR Instability Studies Program for many stimulating discussions, M. Blaskiewicz, K. Harkay, R. Davidson, H. Qin, P. Channell, and T.S. Wang. We are especially grateful to R. Macek for kindly providing us the experimental data and for many valuable discussions. Thanks to R. Kirby, A. Alexandrov, and V. Danilov. We are grateful to NERSC for supercomputer support. This work was supported by the U.S. DOE under Contract No. DEAC03-76SF00098 and by the SNS project (ORNL).

[1] Various contributions in Proceedings of the ICFA Workshop on Two-Stream Instabilities, Santa Fe, NM, 2000, http://www.aps.anl.gov/conferences/icfa/twostream.html; Proceedings of the Two-Stream Instability Workshop, KEK, Tsukuba, 2001 (unpublished) http:// conference.kek.jp/two-stream/; Proceedings of the PAC01, Chicago (IEEE, Piscataway, NJ, 2001) http:// pac2001.aps.anl.gov/; Proceedings of the ECLOUD-02 Workshop, CERN (CERN Yellow Report No. CERN-
2002-001, 2002) http://slap.cern.ch/collective/ecloud02/, especially the surveys by F. Zimmermann, G. Rumolo, M. Blaskiewicz, and R. Macek; Proceedings of the EPAC02, Paris, 2002 (to be published).

[2] R. Macek, in Proceedings of the ECLOUD-02 Workshop, CERN (Ref. [1]).

[3] For an update on the self-consistent treatment of the instability, see contributions to Physical Review Special Topics - Accelerators and Beams Special Collection on Electron Cloud and Two-Stream Interactions in Long-Bunch Proton Beams, http:// prst-ab.aps.org/speced/TwoStreamSC.

[4] K. Ohmi, T. Toyama, and C. Ohmori, Phys. Rev. ST Accel. Beams 5, 114402 (2002).

[5] M. A. Furman and M. Pivi, Phys. Rev. ST Accel. Beams 5, 124404 (2002).

[6] R. E. Kirby and F. K. King, Nucl. Instrum. Methods Phys. Res., Sect. A 469, 1-12 (2001), and private communications.

[7] H. Bruining, Physics and Applications of Secondary Electron Emission (Pergamon Press, McGraw-Hill Book Co., New York, 1954).

[8] P. A. Redhead, J. P. Hobson, and E.V. Kornelsen, The Physical Basis of Ultrahigh Vacuum (Chapman and Hall, London, 1968), Chap. 4 (reprinted by the AIP in 1993 as part of the American Vacuum Society Classics series).

[9] H. Seiler, J. Appl. Phys. 54, R1-R18 (1983).

[10] M. A. Furman and G. R. Lambertson, in Proceedings of the International Workshop on Multibunch Instabilities in Future Electron and Positron Accelerators MBI97 (KEK, Tsukuba, Japan, 1997), KEK Proceedings 9717, edited by Y. H. Chin, p. 170.

[11] E. M. Baroody, Phys. Rev. 78, 780-787 (1950).

[12] V. Baglin, I. Collins, B. Henrist, N. Hilleret, and G. Vorlaufer, CERN LHC Project Report No. 472, 2001 (unpublished).

[13] M. A. Furman, M. Pivi, R. Macek, and A. Browman (to be published).

[14] M. A. Furman, LBNL Report No. LBNL-41482/CBP Note 247, 1998; CERN LHC Project Report No. 180, 1998 (unpublished).

[15] M. Pivi, http://slap.cern.ch/collective/ecloud02/ newschedule.html

[16] R. Macek, in Proceedings of the PAC01, Chicago (Ref. [1]), p. 688.

[17] M. Furman and M. Pivi, in Proceedings of the PACO1, Chicago (Ref. [1]), p. 708.

[18] M. Blaskiewicz, M. Furman, M. Pivi, and R. Macek (to be published).

[19] T. Toyama and K. Ohmi, in Proceedings of the ECLOUD-02 Workshop, CERN (Ref. [1]).

[20] M.T.F. Pivi and M. A. Furman, in Proceedings of the European Particle Accelerator Conference EPAC02, Paris, 2002, http://epac2002.1al.in2p3.fr/, paper WEPDO006.

[21] M. Blaskiewicz, in Proceedings of the ECLOUD-02 Workshop, CERN (Ref. [1]). 